\title{
Approximate simulation of coupled fast and slow reactions for stochastic chemical kinetics
}

\author{
Eric L. Haseltine and James B. Rawlings \\ Department of Chemical Engineering, University of Wisconsin-Madison, Madison, Wisconsin 53706-1691
}

(Received 3 June 2002; accepted 17 July 2002)

\begin{abstract}
Exact methods are available for the simulation of isothermal, well-mixed stochastic chemical kinetics. As increasingly complex physical systems are modeled, however, these methods become difficult to solve because the computational burden scales with the number of reaction events. This paper addresses one aspect of this problem: the case in which reacting species fluctuate by different orders of magnitude. By partitioning the system into subsets of "fast" and "slow" reactions, it is possible to bound the computational load by approximating "fast" reactions either deterministically or as Langevin equations. This paper provides a theoretical background for such approximations and outlines strategies for computing these approximations. Two motivating examples drawn from the fields of particle technology and biotechnology illustrate the accuracy and computational efficiency of these approximations. (C) 2002 American Institute of Physics. [DOI: 10.1063/1.1505860]
\end{abstract}

\section{INTRODUCTION}

Recently, stochastic simulation of chemical kinetics has received an increased amount of attention from the modeling community. ${ }^{1-3}$ Some of the popular methods of solving isothermal, well-mixed kinetics models stem from seminal works by Gillespie. ${ }^{4,5}$ The simple algorithms outlined in these works, the direct method and the first reaction method, permit modeling of microscopic phenomena in terms of discrete, molecular events. As models become progressively more complex, however, these algorithms often become expensive computationally. Some recent efforts have focused upon reducing the computational load. He, Zhang, Chen, and Yang employ a deterministic equilibrium assumption on polymerization reaction kinetics. ${ }^{6}$ Gibson and Bruck refine Gillespie's first reaction algorithm to reduce the required number of random numbers, a technique that works best for systems in which some reactions occur much more frequently than others. ${ }^{7}$ Rao and Arkin demonstrate how to numerically simulate systems reduced by the quasi-steady-state assumption. ${ }^{8}$ This work expands upon ideas by Janssen ${ }^{9,10}$ and Vlad and Pop ${ }^{11}$ who first examined the adiabatic elimination of fast relaxing variables in stochastic chemical kinetics. Gillespie examines two approximate methods for accelerating simulations, but these methods are currently not implementable for complex systems. ${ }^{12}$ Resat, Wiley, and Dixon address systems with reaction rates varying by several orders of magnitude by applying a probability-weighted Monte Carlo approach, but this method increases error in species fluctuations. ${ }^{13}$

In this paper, we propose a new approximation for the simulation of isothermal, well-mixed stochastic chemical kinetics. For systems with species fluctuating by varying orders of magnitude, the largest fluctuating species require the most time to simulate stochastically because exact stochastic simulation techniques scale with the number of reaction events. ${ }^{7}$ We expand upon the idea of a partitioned system ${ }^{8,11}$ and simulation via Gillespie's direct method ${ }^{4,5}$ to construct approximations that reduce the computational burden for simulation of these species. In particular, we partition the system into subsets of "fast" and "slow" reactions. We approximate the "fast" reactions either deterministically or as Langevin equations, and treat the "slow" reactions as stochastic events with time-varying reaction rates. Such approximations can significantly reduce computational load while accurately reconstructing at least the first two moments of the probability distribution for each species.

This paper is organized as follows: First, we examine the theoretical underpinnings of the approximation. Next, we propose a numerical algorithm for performing the simulation, review several practical implementation issues, and propose a further approximation. We then consider two motivating examples. Finally, we critically examine the technique and present conclusions.

\section{STOCHASTIC PARTITIONING}

The key ideas are to (1) model the state of the reaction system using extents of reaction as opposed to molecules of species, and (2) partition the state into subsets of "fast" and "slow" reactions. With these two modeling choices, we can exploit the structure of the chemical master equation, the governing equation for the evolution of the system probability density, by making order of magnitude arguments. We then derive the master equations that govern the "fast" and "slow" reaction subsets. This section outlines these manipulations in greater detail.

We model the state of the system, $x$, using an extent for each irreversible reaction. ${ }^{14}$ An extent of reaction model is consistent with a molecule balance model since

$$
n=n_{0}+\nu^{T} x,
$$

where, assuming that there are $m$ extents of reaction and $p$ chemical species: $x$ is the state of the system in terms of extents (an $m$-vector); $n$ is the number of molecules of 
each species (a $p$-vector); $n_{0}$ is the initial number of molecules of each species (a $p$-vector); and $\nu$ is the stoichiometric matrix (an $m \times p$-matrix).

The upper and lower bounds of $x$ are constrained by the limiting reactant species. We arbitrarily set the initial condition to the origin. Given assumptions outlined by Gillespie, ${ }^{15}$ the governing equations for this system is the chemical master equation,

$$
\frac{d P(x ; t)}{d t}=\sum_{k=1}^{m} a_{k}\left(x-\delta_{k}\right) P\left(x-\delta_{k} ; t\right)-a_{k}(x) P(x ; t),
$$

where $P(x ; t)$ is the probability that the system is in state $x$ at time $t ; a_{k}(x) d t$ is the probability to order $d t$ that reaction $k$ occurs in the time interval $[t, t+d t)$; and $\delta_{k}$ is the $k$ th column of the $(m \times m)$-identity matrix $\delta$. The structure of $\delta$ arises for this particular chemical master equation because the reactions are irreversible.

Now we examine the time scale over which the extents of reaction change. We must first determine a relevant time scale so that we can partition the extents into two subsets: those that have small propensity functions $\left[a_{k}(x)\right.$ 's $]$ and occur few if any times over the time scale, and those that have large propensity functions and occur numerous times over the given time scale. We designate these subsets of $x$ as the $(m-l)$-vector $y$ and the $l$-vector $z$, respectively. Note that

$$
x=\left[\begin{array}{l}
z \\
y
\end{array}\right] \quad \text { and } \delta=\left[\frac{\delta^{z} 0}{0 \delta^{y}}\right]=\left[\begin{array}{c}
\bar{\delta} \\
\bar{\delta}
\end{array}\right]
$$

in which $\delta^{z}$ and $\delta^{y}$ are $(l \times l)$ - and $(m-l \times m-l)$-identity matrices, respectively. Equation (2) becomes

$$
\begin{aligned}
\frac{d P(y, z ; t)}{d t}= & \sum_{k=1}^{m} a_{k}\left(y-\underline{\delta}_{k}, z-\bar{\delta}_{k}\right) P\left(y-\underline{\delta}_{k}, z-\bar{\delta}_{k} ; t\right) \\
& -a_{k}(y, z) P(y, z ; t) .
\end{aligned}
$$

If we can determine the governing equations for the evolution of the marginal probability densities, $P(z ; t)$ and $P(y ; t)$, we then know how the fast and slow reactions evolve over this time scale. Also, this partitioning is similar to that used by Rao and Arkin, ${ }^{8}$ who partition the master equation by species to treat the quasi-steady-state assumption. We partition by reaction extents to treat fast and slow reactions.

\section{A. Fast reaction subset}

First we address the evolution of the marginal probability density for the subset of fast reactions $z$. Following Rao and Arkin, ${ }^{8}$ we define the joint density $P(y, z ; t)$ as the product of the conditional density $P(y \mid z ; t)$ and the desired marginal density $P(z ; t)$,

$$
P(y, z ; t)=P(y \mid z ; t) P(z ; t) .
$$

Differentiating Eq. (5) with respect to time yields

$$
\frac{d P(y, z ; t)}{d t}=\frac{d P(y \mid z ; t)}{d t} P(z ; t)+\frac{d P(z ; t)}{d t} P(y \mid z ; t) .
$$

Over our chosen time scale, it is highly improbable that any reactions in subset $y$ will occur. Using order of magnitude arguments, Eq. (6) becomes

$$
\frac{d P(y, z ; t)}{d t} \approx \frac{d P(z ; t)}{d t} P(y \mid z ; t) .
$$

Manipulating Eq. (7) yields

$$
\begin{aligned}
\frac{d P(z ; t)}{d t}= & \sum_{y} \sum_{k=1}^{m} a_{k}\left(y-\underline{\delta}_{k}, z-\bar{\delta}_{k}\right) \\
& \times P\left(y-\underline{\delta}_{k} \mid z-\bar{\delta}_{k} ; t\right) P\left(z-\bar{\delta}_{k} ; t\right) \\
& -a_{k}(y, z) P(y \mid z ; t) P(z ; t) .
\end{aligned}
$$

By exploiting the structure of $\delta$ (an identity matrix), we solve for the desired derivative,

$$
\frac{d P(z ; t)}{d t}=\sum_{k=1}^{l} b_{k}\left(z-\delta_{k}^{z}\right) P\left(z-\delta_{b}^{z} ; t\right)-b_{k}(z) P(z ; t),
$$

in which

$$
b_{k}(z)=\sum_{y} a_{k}(y, z) P(y \mid z ; t) .
$$

If the reaction propensities $a_{k}(y, z)$ in Eq. (10) are linear with respect to $y$, then the following equation holds:

$$
b_{k}(z)=a_{k}(E[y \mid z], z) .
$$

If the reaction propensities are nonlinear with respect to $y$, Eq. (11) still provides a first order approximation to Eq. (10). Also, note that Eqs. (5)-(10) are the fast/slow reaction analogs of the quasi-steady-state results developed by Rao and Arkin. ${ }^{8}$

With the resulting partition, we are now in a position to better handle the fast subset of reactions $z$. We proceed by demonstrating as outlined by Gardiner ${ }^{16}$ how this subset can be approximated using the Langevin approximation. Define the characteristic size of the system to be $\Omega$, and use this size to recast the master Eq. (9) in terms of intensive variables (let $\bar{z} \leftarrow z / \Omega$ ). Performing a Kramers-Moyal expansion on this master equation results in a system size expansion in $\Omega$. In the limit as $z$ and $\Omega$ becomes large, the discrete master equation can be approximated by its first two differential moments with the continuous Fokker-Planck equation,

$$
\begin{aligned}
\frac{\partial P(z ; t)}{\partial t}= & -\sum_{i=1}^{l} \frac{\partial}{\partial z_{i}} A_{i}(z) P(z ; t) \\
& +\frac{1}{2} \sum_{i=1}^{l} \sum_{j=1}^{l} \frac{\partial^{2}}{\partial z_{i} \partial z_{j}}\left[B_{i j}(z)\right]^{2} P(z ; t),
\end{aligned}
$$

in which (noting that $z$ consists of extents of reaction),

$$
\begin{gathered}
A(z)=\sum_{i=1}^{l} \delta_{i}^{z} b_{i}(z)=\left[b_{1}(z) b_{2}(z) \cdots b_{l}(z)\right]^{T}, \\
{[B(z)]^{2}=\sum_{i=1}^{l} \delta_{i}^{z}\left(\delta_{i}^{z}\right)^{T} b_{i}(z)} \\
=\operatorname{diag}\left(b_{1}(z), b_{2}(z), \ldots, b_{l}(z)\right) .
\end{gathered}
$$

Equation (12) has Itô solution of the form, 


$$
\begin{aligned}
d z_{i} & =A_{i}(z) d t+\sum_{j=1}^{l} B_{i j}(z) d W_{j} \\
& =b_{i}(z) d t+\sqrt{b_{i}(z)} d W_{i}
\end{aligned}
$$

in which $W$ is the Wiener process. Equation (15) is the chemical Langevin equation, whose formulation was recently addressed by Gillespie. ${ }^{17}$ Note the difference between Eqs. (12) and (15). The Fokker-Planck Eq. (12) specifies the distribution of the stochastic process, whereas the stochastic differential Eq. (15) specifies how the trajectories of the state evolve. Also, bear in mind that whether or not a given $\Omega$ is large enough to permit truncation of the system size expansion is relative. In this case, $\Omega$ is of sufficient magnitude to make this approximation valid for only a subset of the reactions, not the entire system.

\section{B. Slow reaction subset}

Since the subset of slow reaction extents $y$ is a function of the fast reaction extents $z$, the problem of interest is a master equation subject to constraints,

$$
\begin{aligned}
\frac{d P(y ; t)}{d t}= & \sum_{k=l+1}^{m} a_{k}\left(y-\underline{\delta}_{k}, z ; t\right) P\left(y-\underline{\delta}_{k} ; t\right) \\
& -a_{k}(y, z ; t) P(y ; t) \\
\text { s.t.: } d z_{i}= & b_{i}(z) d t+\sqrt{b_{i}(z)} d W_{i}, \quad i=1, \ldots, l .
\end{aligned}
$$

Gillespie $^{18}$ outlines a general method for exact stochastic simulation of time dependent propensities that is applicable for solution of the desired problem, Eq. (16). This method examines the joint probability function, $P(\tau, \mu)$, that governs when the next reaction occurs, and which reaction occurs. Conditioning $P(\tau, \mu)$ indicates that the probability of a reaction occurring in time $\tau$ is

$$
P(\tau)=r_{\text {tot }}^{y}(t+\tau) \exp \left(-\int_{t}^{t+\tau} r_{\text {tot }}^{y}\left(t_{1}\right) d t_{1}\right)
$$

and the probability that reaction $\mu$ occurs is

$$
P(\mu \mid \tau)=\frac{a_{\mu}(y, z ; t+\tau)}{r_{\mathrm{tot}}^{y}(t+\tau)}
$$

in which

$$
r_{\mathrm{tot}}^{y}(t)=\sum_{k=l+1}^{m} a_{k}(y, z ; t) .
$$

\section{NUMERICAL IMPLEMENTATION}

We now outline a procedure for implementing the approximation of the previous section. Using Monte Carlo simulation, we obtain realizations of the desired joint probability function $P(\tau, \mu)$ by randomly selecting $\tau$ and $\mu$ from the probability densities defined by Eqs. (17) and (18). Given two random numbers $p_{1}$ and $p_{2}$ uniformly distributed on $(0,1), \tau$ and $\mu$ are constrained accordingly,

$$
\int_{t}^{t+\tau} r_{\mathrm{tot}}^{y}\left(t_{1}\right) d t_{1}+\log \left(p_{1}\right)=0,
$$

$$
\sum_{k=l+1}^{\mu-1} a_{k}(y, z ; t+\tau)<p_{2} r_{\mathrm{tot}}^{y}(t+\tau) \leqslant \sum_{k=l+1}^{\mu} a_{k}(y, z ; t+\tau) .
$$

Since $r_{\text {tot }}^{y}$ is a function of $t$,

$$
\begin{aligned}
& \frac{d r_{\mathrm{tot}}^{y}(t)}{d t}=\frac{\partial r_{\mathrm{tot}}^{y}(t)}{\partial z^{T}} \frac{d z}{d t}, \\
& r_{\mathrm{tot}}^{y}\left(t_{1}\right)=\int_{t}^{t_{1}} \frac{d r_{\mathrm{tot}}^{y}\left(t_{2}\right)}{d t_{2}} d t_{2} .
\end{aligned}
$$

Hence for practical implementation, we satisfy Eq. (20a) by integrating $r_{\text {tot }}^{y}$ and the fast subset of reactions $z$ forward in time until the following condition is met,

$$
\int_{t}^{t+\tau} \int_{t}^{t_{1}} \frac{d r_{\mathrm{tot}}^{y}\left(t_{2}\right)}{d t_{2}} d t_{2} d t_{1}+\log \left(p_{1}\right)=0 .
$$

We now propose an algorithm for solving the partitioned reaction system.

Off-line: Determine the criteria for when and how the set $x$ of $m$ extents of reaction should be partitioned. Determine the stoichiometric matrices of the form given in Eq. (1) and reaction propensity laws for the unpartitioned [the $(m \times p)$ matrix $\nu$ and $a_{k}(x)$ 's] and partitioned cases [the $(m-l \times p)$ matrix $\nu^{y}$, the $(l \times p)$-matrix $\nu^{z}$, and $a_{k}^{y}(y, z)$ 's]. Also, determine the necessary Langevin equations in the form of Eq. (15) for the fast reactions in the partitioned case.

Initialize: Set the time, $t$, equal to zero.

Set the number of species $n$ to $n_{0}$.

(1) If the partitioning criteria established off-line are met, go to step (5).

(2) Calculate: (a) the reaction propensities, $r_{k}=a_{k}(x)$, and (b) the total reaction propensity, $r_{\text {tot }}=\sum_{k=1}^{m} r_{k}$.

(3) Select two random numbers $p_{1}, p_{2}$ from the uniform distribution $(0,1)$. Let $\tau=-\log \left(p_{1}\right) / r_{\text {tot }}$. Choose $j$ such that

$$
\sum_{k=1}^{j-1} r_{k}<p_{2} r_{\mathrm{tot}} \leqslant \sum_{k=1}^{j} r_{k}
$$

(4) Let $t \leftarrow t+\tau$. Let $n \leftarrow n+\nu_{j}^{T}$, where $\nu_{j}$ is the $j$ th row of $\nu$. Go to step (1).

(5) For subset $y$, calculate (a) the reaction propensities, $r_{k}^{y}=a_{k}^{y}(y, z)$, and (b) the total reaction propensity, $r_{\text {tot }}^{y}$ $=\sum_{k=1}^{m-l} r_{k}^{y}$.

(6) Select two random numbers $p_{1}, p_{2}$ from the uniform distribution $(0,1)$.

(7) Determine $\hat{\nu}_{z}=\left(\nu^{z}\right)^{T}[z(t+\tau)-z(t)]$ by integrating $r_{\text {tot }}^{y}(t)$ and the subset of fast reactions $z$ until the following condition is met:

$$
\begin{aligned}
& \int_{t}^{t+\tau} \int_{t}^{t_{1}} \frac{d r_{\mathrm{tot}}^{y}\left(t_{2}\right)}{d t_{2}} d t_{2} d t_{1}+\log \left(p_{1}\right)=0 \\
& \text { s.t.: } \quad r_{\mathrm{tot}}^{y}(t)=\sum_{k=1}^{m-l} a_{k}^{y}(y, z ; t) .
\end{aligned}
$$

(8) Let $t \leftarrow t+\tau$. Let $n \leftarrow n+\hat{\nu}_{z}$.

(9) Choose $j$ such that ${ }^{19}$ 


$$
\sum_{k=1}^{j-1} r_{k}^{y}<p_{2} r_{\text {tot }}^{y}(t) \leqslant \sum_{k=1}^{j} r_{k}^{y} .
$$

(10) Let $n \leftarrow n+\left(\nu_{j}^{y}\right)^{T}$, where $\nu_{j}^{y}$ is the jth row of $\nu^{y}$. Go to step (1).

Over the time interval $\tau$, implementation of this algorithm actually enforces the more stringent requirement that

$$
\frac{d P(y \mid z)}{d t}=0
$$

Hence Eq. (7) is exact, not approximate.

\section{A FURTHER APPROXIMATION: SCALING THE STOCHASTIC TIME STEP} straint

One major difficulty in this method is satisfying the con-

$$
\int_{t}^{t+\tau} r_{\mathrm{tot}}^{y}\left(t_{1}\right) d t_{1}+\log \left(p_{1}\right)=0
$$

in step (7) of the proposed numerical algorithm as opposed to the simple algebraic relation for $\tau$ used in the unmodified Gillespie algorithm [i.e., step (3)]. This constraint can prove to be computationally expensive.

If the reaction propensities for the fast subset of extents $z$ change insignificantly over the stochastic time step $\tau$, the unmodified Gillespie algorithm can still provide an approximate solution. When the reaction propensities change significantly over $\tau$, steps can be taken to reduce the error of the Gillespie algorithm. One idea is to scale the stochastic time step $\tau$ by artificially introducing a probability of no reaction into the system. Let $a_{0} d t$ be the contrived probability, first order in $d t$, that no reaction occurs in the next time interval $d t$. This probability does not effect the number of molecules of the modeled reaction system while allowing adjustment of the stochastic time step by changing the magnitude of $a_{0}$. Theoretically, as the magnitude of $a_{0}$ becomes infinite, the total reaction rate becomes infinite. As the total reaction rate approaches infinity, the error of the stochastic simulation subject to constraints approaches zero because the algorithm checks whether or not a reaction occurs at every time. Even though the method outlined by Gillespie ${ }^{18}$ and Jansen ${ }^{20}$ is "exact," for this case there is still error associated with (1) the number of simulations performed since it is a Monte Carlo method, and (2) integration of the Langevin equations for the fast extents of reaction. Thus it is plausible that these errors may be greater than the error introduced by the approximation. Hence our approximation may often prove to be less computationally expensive than the exact solution while generating an acceptable amount of simulation error.

The approximation modifies steps (5)-(10) of the numerical algorithm as follows:

(5) For subset $y$, calculate (a) the reaction propensities, $r_{k}^{y}=a_{k}^{y}(y, z)$, and (b) the total reaction propensity, $r_{\text {tot }}^{y}$ $=\sum_{k=0}^{m-l} r_{k}^{y}$.

(6) Select two random numbers $p_{1}, p_{2}$ from the uniform distribution $(0,1)$.
(7) Let $\tau=-\log \left(p_{1}\right) / r_{\text {tot }}$. Integrate subset $z$ over the range $[t, t+\tau)$ to determine $\hat{\nu}_{z}=\left(\nu^{z}\right)^{T}[z(t+\tau)-z(t)]$. Let $t \leftarrow t+\tau$. Let $n \leftarrow n+\hat{\nu}_{z}$.

(8) Recalculate the reaction propensities $r_{k}^{y}$ 's and the total reaction propensity $r_{\text {tot }}^{y}(t)$. Choose $j$ such that

$$
\sum_{k=0}^{j-1} r_{k}^{y}<p_{2} r_{\text {tot }}^{y}(t) \leqslant \sum_{k=0}^{j} r_{k}^{y} .
$$

(9) Let $n \leftarrow n+\left(\nu_{j}^{y}\right)^{T}$, where $\nu_{j}^{y}$ is the jth row of $\nu^{y}$. Go to step (1).

\section{PRACTICAL IMPLEMENTATION}

Partitioning of the state $x$ into "fast" and "slow" extents should be intuitive. We recommend maintaining at least two orders of magnitude difference between the values of the partitioned reaction probabilities. It may also be helpful to generate results for a full stochastic simulation, and then identify which reactions are bottlenecks (i.e., ones occurring most frequently). Note that there may exist several regimes that require different partitioning of the state. Also, care should be exercised to maintain the validity of the order of magnitude partition between $y$ and $z$. It is obviously undesirable for "slow" reaction extents to become the same order of magnitude of the "fast" extents during the time increment $\tau$.

In the thermodynamic limit $(z \rightarrow \infty, \Omega \rightarrow \infty, \bar{z}=z / \Omega$ $=$ finite), the intensive variables for the fast subset of reactions ( $\bar{z}$ 's) evolve deterministically. ${ }^{21}$ Accordingly, we propose approximating the Langevin Eq. (15) with

$$
\frac{d z_{i}(t)}{d t}=b_{i}(z(t)) \text {. }
$$

The benefit of this assumption is that Eq. (26) can be solved rigorously using an ODE solver. Unfortunately for physical systems, the thermodynamic limit is obviously unattainable. However, knowledge of the modeled system can lead to this simplification. If the magnitude of the fluctuations in this term is small compared to the sensitivity of $b_{i}(z(t))$ to the subset $y$, then Eq. (26) is a valid approximation. This approximation is also valid if one is primarily concerned with the fluctuations in the small-numbered species as opposed to the large-numbered species, assuming that the extents approximated by Eq. (26) predominantly affect the population size of large-numbered species.

\section{EXAMPLES}

We now consider two motivating examples that illustrate the accuracy of the presented stochastic approximations. For clarity, we first briefly review the nomenclature that indicates which approximations, if any, are performed in a given simulation. We can either perform a purely stochastic simulation on the unpartitioned reaction system, or we can partition the system into "fast" and "slow" reactions. For this partitioned case, a stochastic-Langevin simulation treats the fast reactions as Langevin equations, while a stochastic-deterministic simulation treats the fast reactions deterministically. We can then simulate this partitioned reaction system by exact simulation, in which the next reaction time exactly accounts for the time dependence of the "fast" reactions upon the "slow" 
TABLE I. Model parameters and reaction extents for the simple crystallization example.

\begin{tabular}{lcl}
\hline \hline \multicolumn{1}{c}{ Parameter } & Symbol & \multicolumn{1}{c}{ Value } \\
\hline Extent of reaction (27a) & $\epsilon_{1}$ & $\frac{1}{2} k_{1} \mathrm{~A}(\mathrm{~A}-1)$ \\
Extent of reaction (27b) & $\epsilon_{2}$ & $k_{2} \mathrm{AC}$ \\
Reaction (27a) rate constant & $k_{1}$ & $1 \mathrm{E}-7$ \\
Reaction (27b) rate constant & $k_{2}$ & $1 \mathrm{E}-7$ \\
Initial number of A molecules & $\mathrm{A}_{o}$ & $1 \mathrm{E}+6$ \\
Initial number of B molecules & $\mathrm{B}_{o}$ & 0 \\
Initial number of C molecules & $\mathrm{C}_{o}$ & 10 \\
Initial number of D molecules & $\mathrm{D}_{o}$ & 0 \\
\hline \hline
\end{tabular}

reactions; or by an approximate simulation, which neglects this time dependence but scales the next reaction time with a propensity of no reaction.

\section{A. Simple crystallization}

Consider a simplified reaction system for the crystallization of species A:

$$
\begin{aligned}
& 2 \mathrm{~A} \stackrel{k_{1}}{\longrightarrow} \mathrm{B} \epsilon_{1}, \\
& \mathrm{~A}+\mathrm{C} \stackrel{k_{2}}{\longrightarrow} \mathrm{D} \epsilon_{2} .
\end{aligned}
$$

The model parameters and the reaction extents are given in Table I. For this example, the first reaction occurs many more times than the second reaction. Hence we partition the extents of reaction ( $\epsilon_{i}$ 's) as follows: ${ }^{22} \epsilon_{2}$ comprises the subset of slow reactions $y$; and $\epsilon_{1}$ comprises the subset of fast reactions $z$.

We first perform an approximate stochastic-Langevin simulation. Here we approximate the fast reaction subset using the Langevin approximation and attempt to reconstruct the first two moments of each species. The Langevin equations are integrated using the Euler-Murayama method ${ }^{23}$ with a time increment of 0.01 . We account for the timevarying propensity of the slow reaction by employing the approximate scheme, setting the propensity of no reaction $\left(a_{0}\right)$ to 10 . Figure 1 compares these results to the exact stochastic results for 10000 simulations. The approximation accurately reconstructs the mean and standard deviation for all species.

Next, we approximate the fast reaction subset deterministically and attempt to reconstruct the first two moments of each species based upon 10000 simulations. For this case, we consider both the exact and approximate stochasticdeterministic simulations.

Figure 2 compares the results of exact stochastic simulation to the exact stochastic-deterministic solution. This ap-
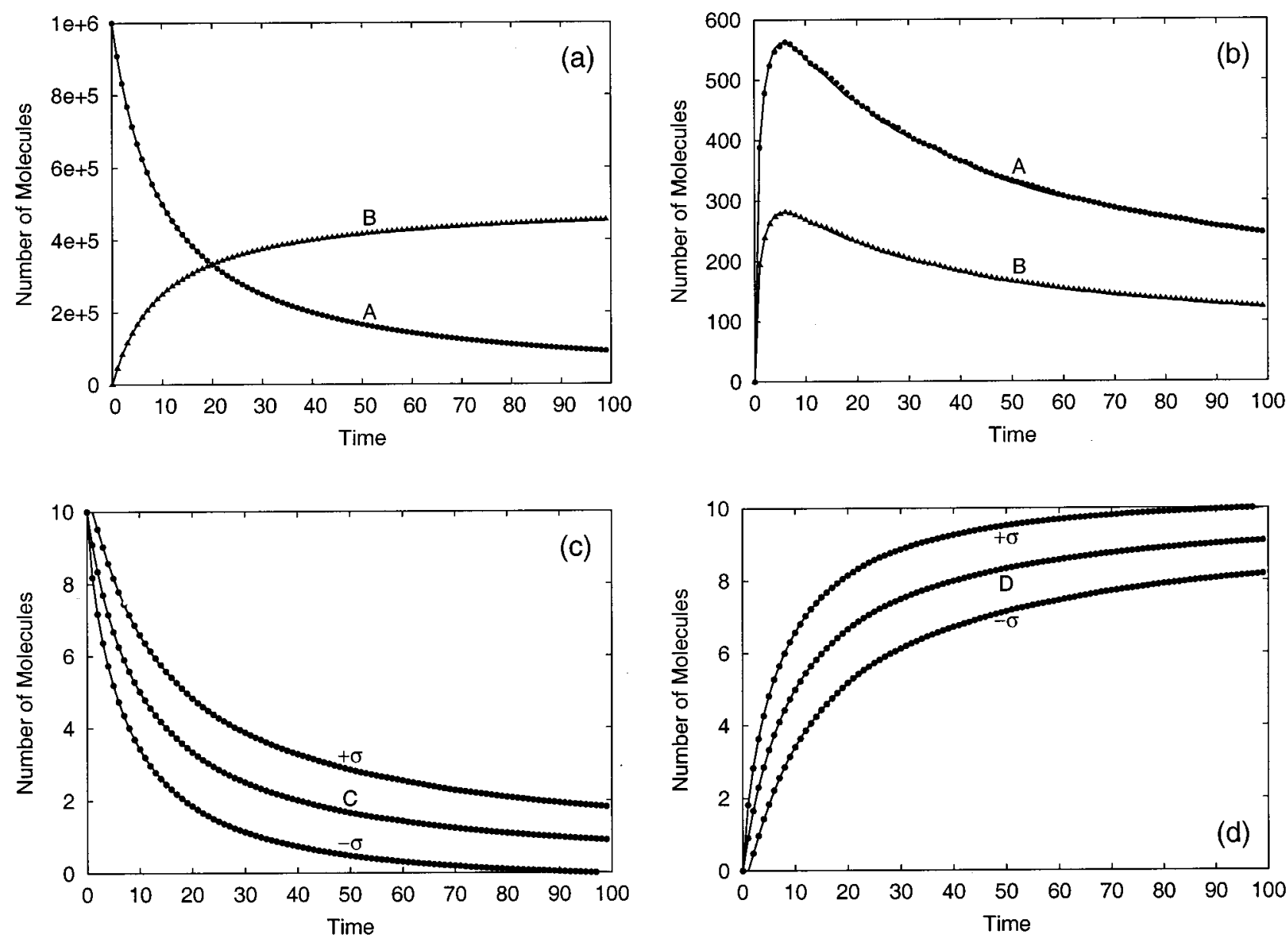

FIG. 1. Comparison of approximate stochastic-Langevin simulation (points) to exact stochastic simulation (lines) based on 10000 simulations, propensity of no reaction $a_{0}=10$, and Langevin time step of 0.01. (a) Compares the mean for species A and B. (b) Compares the standard deviations for species A and B. (c) Compares the mean (C) and standard deviation $( \pm \sigma)$ for species C. (d) Compares the mean (D) and standard deviation ( $\pm \sigma)$ for species D. 

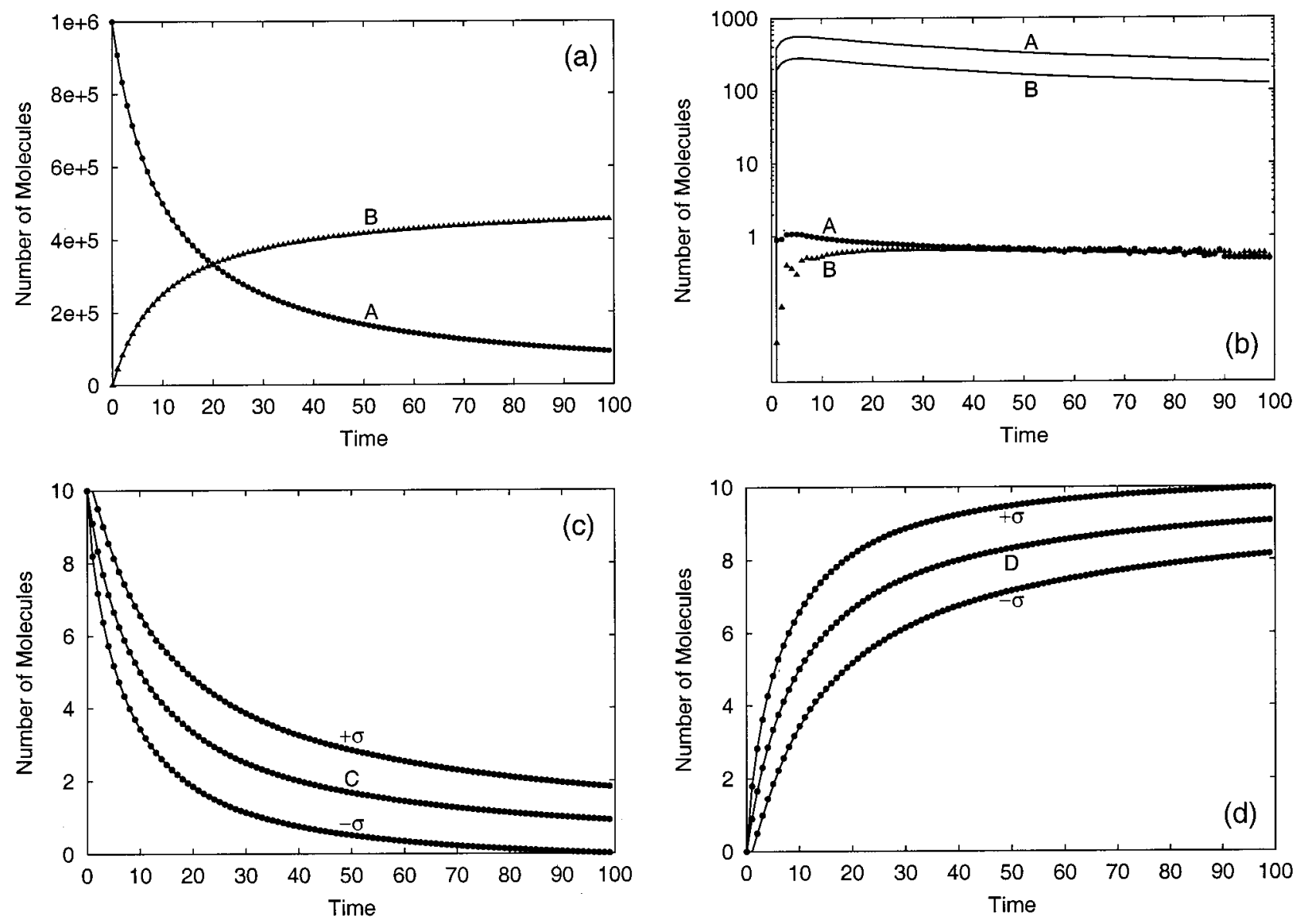

FIG. 2. Comparison of exact stochastic-deterministic simulation (points) to exact stochastic simulation (lines) based on 10000 simulations. (a) Compares the mean for species A and B. (b) Compares the standard deviations for species A and B. (c) Compares the mean (C) and standard deviation ( $\pm \sigma$ ) for species C. (d) Compares the mean (D) and standard deviation $( \pm \sigma)$ for species D.

proximation does an excellent job of reconstructing all of the means as well as the standard deviations for species $\mathrm{C}$ and $\mathrm{D}$. However, we are not able to reconstruct the standard deviations for species A and B. This phenomenon is expected because by approximating $\epsilon_{1}$ deterministically, we neglect all fluctuations caused by the first reaction.

Figure 3 compares the results of exact stochastic simulation to the approximate stochastic-deterministic solution given a small value for the propensity of no reaction, $a_{0}$. For this value of $a_{0}$, the approximation accurately reconstructs the means of species $\mathrm{A}$ and $\mathrm{B}$, but fails to reconstruct the moments of species $\mathrm{C}$ and $\mathrm{D}$ as well as the standard deviations of species $\mathrm{A}$ and $\mathrm{B}$. This phenomenon indicates that the value of $a_{0}$ is too small. By examining the cumulative squared error, however, Fig. 4 demonstrates that increasing the value of $a_{0}$ results in comparable error for the approximate and exact stochastic-deterministic simulations. Here, the least squares error is based on the deviation of the species $\mathrm{C}$ trajectories between the approximation techniques and the exact stochastic simulation.

Table II compares the order of magnitude of the limiting time step for the different methods in this example. The major improvement in the approximate methods is that the time step is now limited by the "slow" reaction time as opposed to the "fast" reaction time. Note that the solution methods for the partitioned reaction system require more computa- tional expense per limiting time step than the exact stochastic solution method. However, we still observed an order of magnitude improvement in computational expense by employing the approximate solution methods.

\section{B. Intracellular viral infection}

We now consider a general model of an infection of a cell by a virus. A reduced system model consists of the following reaction mechanism: ${ }^{24}$

$$
\begin{aligned}
& \text { nucleotides } \stackrel{\text { template }}{\longrightarrow} \text { genome } \epsilon_{1}, \\
& \text { nucleotides }+ \text { genome } \longrightarrow \text { template } \epsilon_{2}, \\
& \text { nucleotides }+ \text { amino acids } \stackrel{\text { template }}{\longrightarrow} \text { struct } \epsilon_{3}, \\
& \text { template } \longrightarrow \text { degraded } \epsilon_{4}, \\
& \text { struct } \longrightarrow \text { secreted/degraded } \epsilon_{5}, \\
& \text { genome }+ \text { struct } \longrightarrow \text { secreted virus } \epsilon_{6},
\end{aligned}
$$

where genome and template are the genomic and template viral nucleic acids, respectively, and struct is the viral structural protein. Additional assumptions include: (1) nucleotides and amino acids are available at constant concentrations, and (2) template catalyzes reactions (28a) and (28c). 

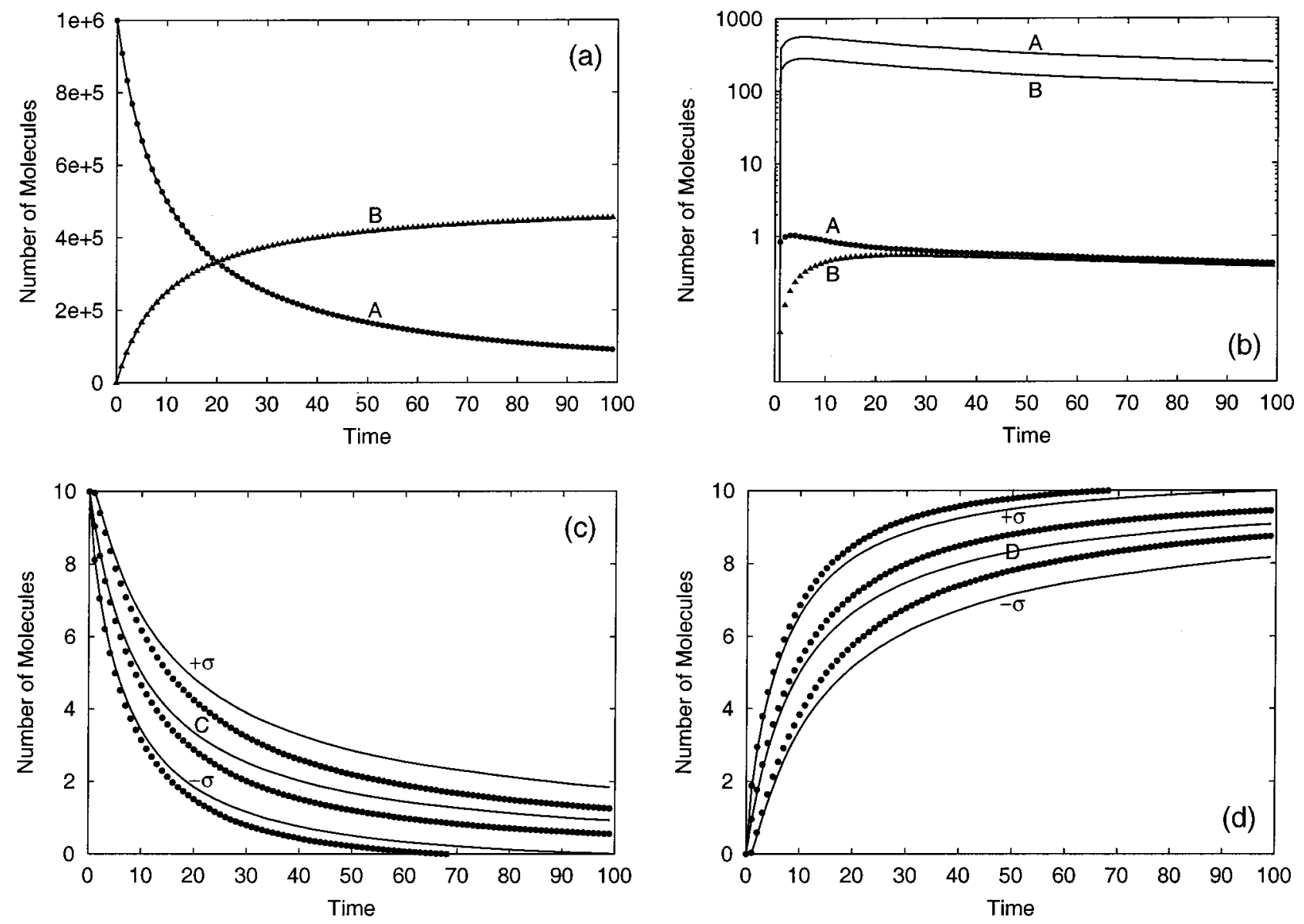

FIG. 3. Comparison of approximate stochastic-deterministic simulation (points) to exact stochastic simulation (lines) based on 10000 simulations and propensity of no reaction $a_{0}=0.01$. (a) Compares the mean for species A and B. (b) Compares the standard deviations for species A and B. (c) Compares the mean (C) and standard deviation $( \pm \sigma)$ for species C. (d) Compares the mean (D) and standard deviation $( \pm \sigma)$ for species D.

We are interested in the time evolution of the template, genome, and struct species. We assume that the initial "infection" of a cell corresponds to the insertion of one template molecule into the cell. The model parameters and reaction extents are presented in Table III.

This model has two interesting features best illustrated by the two exact stochastic simulations presented in Fig. 5 . First, the three components of the model exhibit fluctuations that vary by differing orders of magnitude. For the same time scale, the struct species fluctuates by hundreds to thousands of molecules, whereas the template and genome species fluctuate by tens of molecules. Second, the model solution exhibits a bimodal distribution. In particular, a cell may exhibit either a "typical" infection in which all species become populated, or an "aborted" infection in which all species are eliminated from the cell.

When the number of template and struct molecules are $>0$ and 100, respectively, reactions (28c) and (28e) occur many more times than the remaining reactions. Hence when template $>0$ and struct $>100$, we partition the system as follows: $\epsilon_{1}, \epsilon_{2}, \epsilon_{4}$, and $\epsilon_{6}$ comprise the subset of slow reactions $y$; and $\epsilon_{3}$ and $\epsilon_{5}$ comprise the subset of fast reactions $z$.

Figure 5 indicates that the simulation should traverse between the partitioned and unpartitioned reaction systems. Since our approximation makes fast reactions continuous events as opposed to discrete ones, we round all species when transitioning from the approximate to exact stochastic simulation to prevent noninteger values. This rounding only affects the struct species, and therefore introduces negligible error into the system.

We choose to approximate the fast reaction subset deterministically, so we employ the approximate stochasticdeterministic simulation with propensity of no reaction $a_{0}$ $=0$. We compare the approximate stochastic-deterministic simulation to the exact stochastic simulation by reconstruct-

TABLE II. Comparison of time steps for the simple crystallization example.

\begin{tabular}{|c|c|c|c|c|}
\hline Solution method & System type & Limiting time step & $O$ (time step) & Relative CPU time \\
\hline Exact stochastic & Unpartitioned & Fast reaction time & $O(1 \mathrm{E}-5)$ & 30 \\
\hline Stochastic-Langevin & Partitioned & Slow reaction time (Langevin integration) & $O(0.01)$ & 1.28 \\
\hline Stochastic-deterministic & Partitioned & Slow reaction time (ODE solver) & $O(1)$ & 1 \\
\hline
\end{tabular}



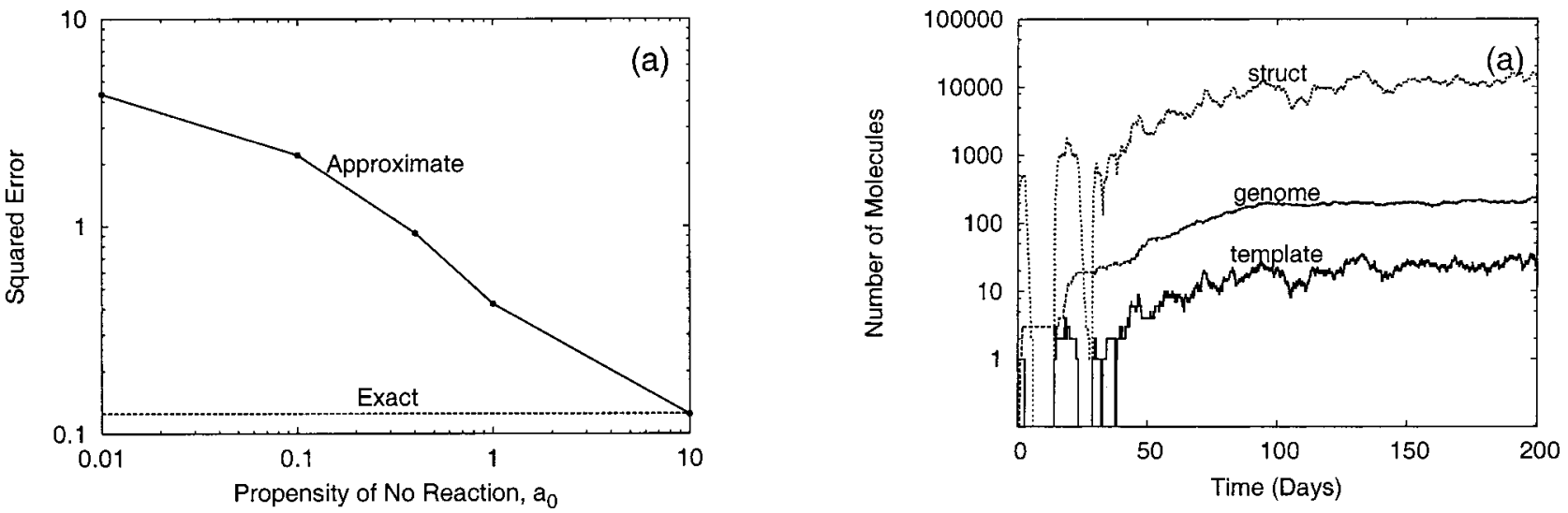

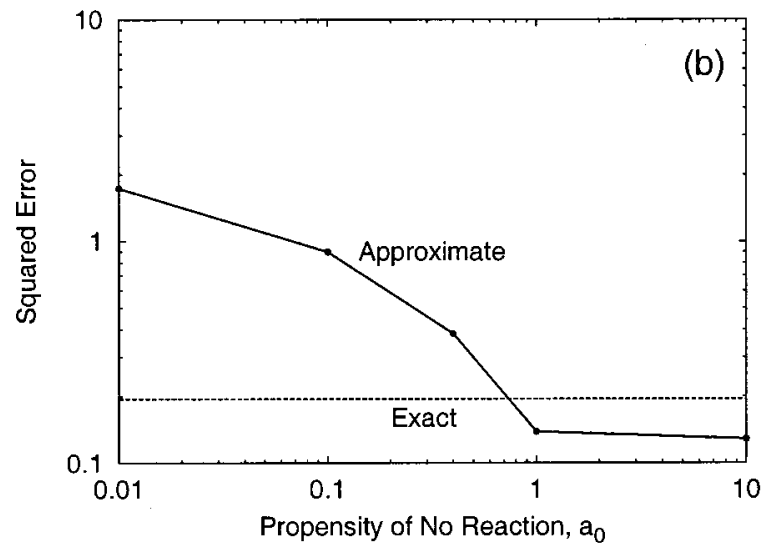

FIG. 4. Squared error trends for the exact and approximate stochasticdeterministic simulations based on 10000 simulations. The squared error is calculated from the deviation of the moments for species $\mathrm{C}$ between the approximation techniques and the exact stochastic simulation. (a) Plots the error in the mean of species C. (b) Plots the error in the standard deviation of species $\mathrm{C}$.

ing the statistics for each species based upon 1000 simulations. We also compare the evolution of the mean for these two simulations to the solution of the purely deterministic model.

Figures 6-8 compare the time evolution of the probability distribution for the template, the small numbered species.

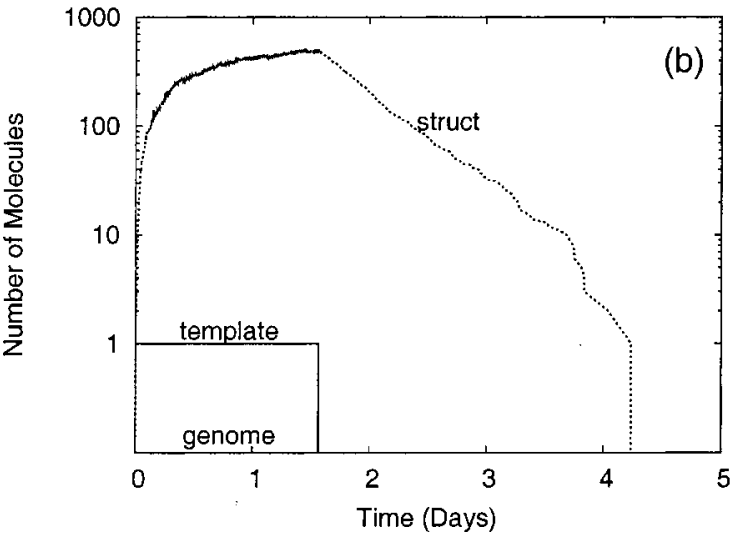

FIG. 5. (a) Typical and (b) aborted intracellular viral infections.

These figures indicate that the approximate stochasticdeterministic simulation accurately reconstructs the entire template probability distribution. Note that the purely deterministic model, however, is unable to accurately reconstruct even the evolution of the mean. This phenomena occurs because the deterministic model cannot describe the bimodal nature of the probability density.

Figure 9 compares the evolution of the mean and standard deviation for the genome species. Again, the approxi-

TABLE III. Model parameters and reaction extents for the intracellular viral infection example.

\begin{tabular}{lcc}
\hline \hline \multicolumn{1}{c}{ Parameter } & Symbol & Value \\
\hline Extent of reaction (28a) & $\epsilon_{1}$ & $k_{1}$ (template) \\
Extent of reaction (28b) & $\epsilon_{2}$ & $k_{2}$ (genome) \\
Extent of reaction (28c) & $\epsilon_{3}$ & $k_{3}$ (template) \\
Extent of reaction (28d) & $\epsilon_{4}$ & $k_{4}$ (template) \\
Extent of reaction (28e) & $\epsilon_{5}$ & $k_{5}$ (struct) \\
Extent of reaction (28f) & $\epsilon_{6}$ & $k_{6}$ (genome) (struct) \\
Reaction (28a) rate constant & $k_{1}$ & 1 day $^{-1}$ \\
Reaction (28b) rate constant & $k_{2}$ & 0.025 day $^{-1}$ \\
Reaction (28c) rate constant & $k_{3}$ & 1000 day $^{-1}$ \\
Reaction (28d) rate constant & $k_{4}$ & 0.25 day $^{-1}$ \\
Reaction (28e) rate constant & $k_{5}$ & 1.9985 day $^{-1}$ \\
Reaction (28f) rate constant & $k_{6}$ & 1 \\
Initial number of template molecules & template & 0 \\
Initial number of genome molecules & genome $_{o}$ & 0 \\
Initial number of struct molecules & struct $_{o}$ & $0.5 \mathrm{E}-6$ (molecules day $^{-1}$ \\
\hline \hline
\end{tabular}



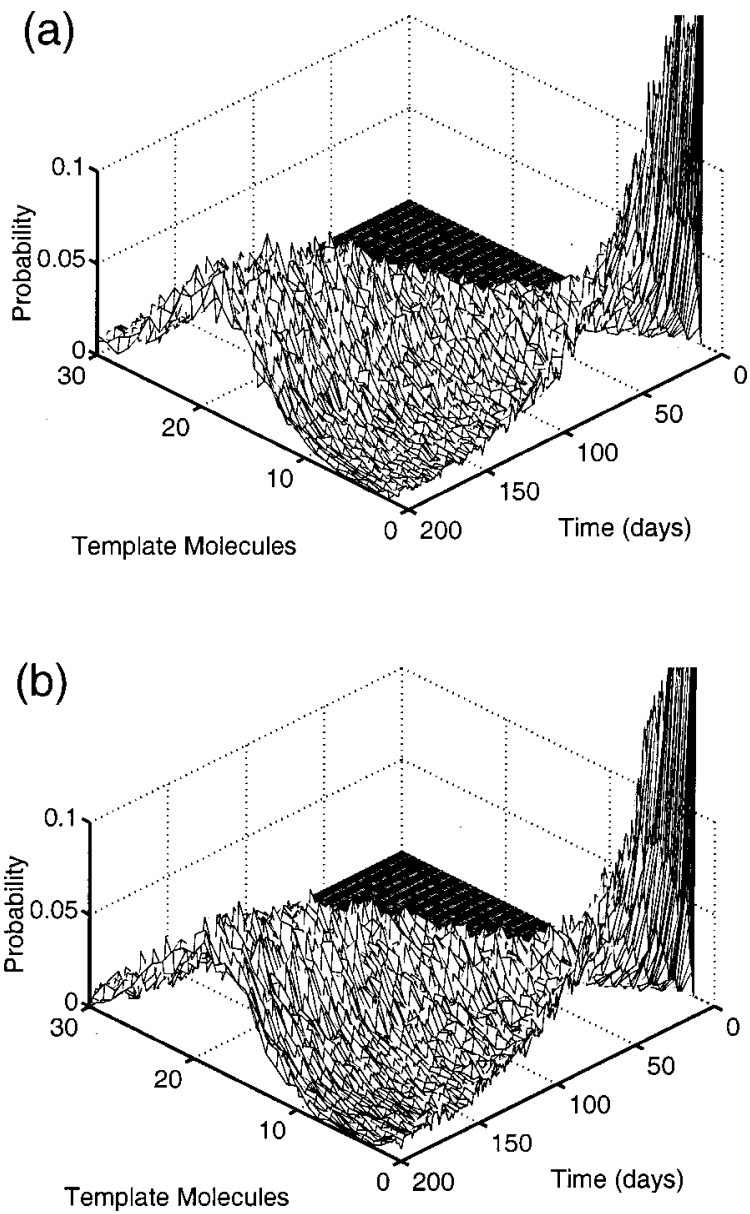

FIG. 6. Evolution of the template probability distribution for the (a) exact stochastic and (b) approximate stochastic-deterministic simulations.

mate simulation accurately reconstructs the time evolution of these moments.

Figure 10 compares the evolution of the mean and standard deviation for struct, the large numbered species. Surprisingly, the approximate stochastic-deterministic simulation accurately reconstructs the time evolution of both of these statistics. Since we approximated the fast reactions deterministically, we did not expect to accurately reconstruct moments higher than the mean for the large numbered species. For this example, though, fluctuations in the small numbered species, template, are amplified into the struct species via reaction $(28 \mathrm{c})$. Thus we are able to accurately reconstruct moments of order higher than zero.

Table IV compares the computational expense between the exact stochastic and approximate stochastic-deterministic solution methods. The approximate solution method results in a 50-fold reduction in computational expense over the exact solution method.

\section{CRITICAL ANALYSIS OF THE STOCHASTIC APPROXIMATIONS}

The primary contribution of this work is the idea of partitioning a purely stochastic reaction system using extents of reaction into subsets of slow and fast reactions. By approximating the fast reaction subset using Langevin equations, we can reduce the computational requirement by integrating the
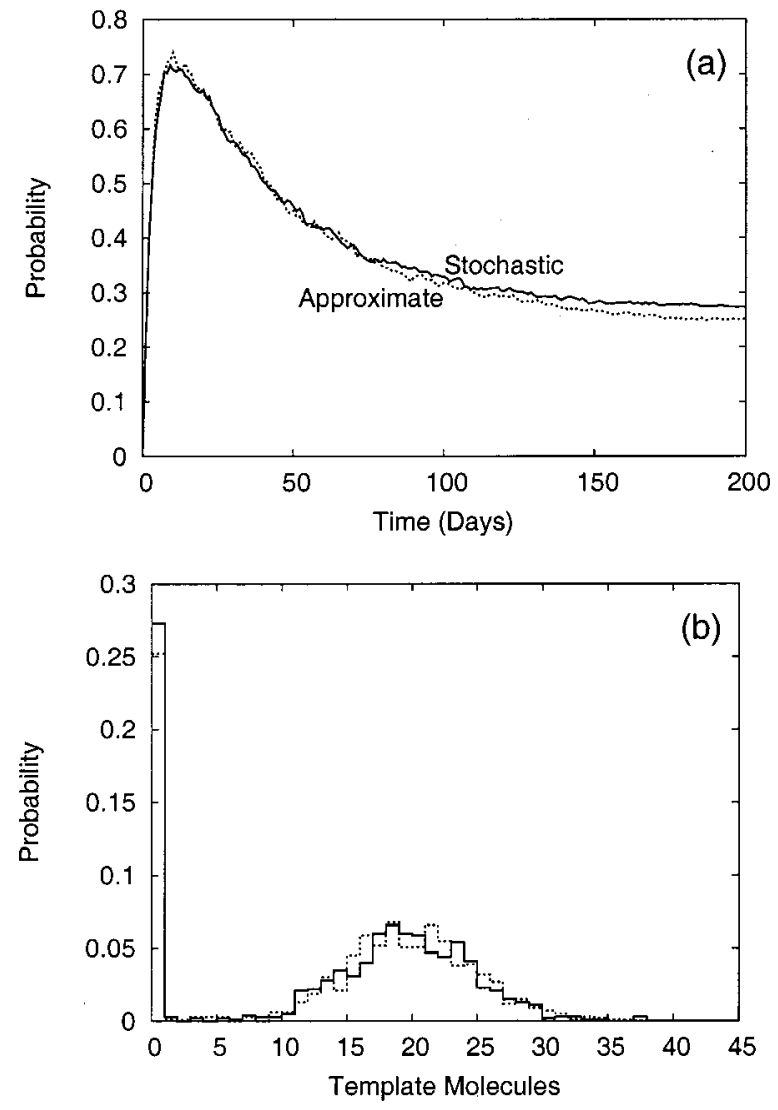

FIG. 7. Comparisons of the (a) (template $=0, t$ ) and (b) (template, $t=200$ days) cross sections of the template probability distribution for the exact stochastic (solid line) and approximate stochastic-deterministic (dashed line) simulations.

system over a much larger time step than the exact stochastic simulation. However, we must now employ schemes for integrating stochastic differential equations. By approximating the fast reaction subset deterministically, we can bound the computational requirements for simulation of the system. For this case, we can employ existing and robust ordinary differential equation solvers for integration of this reaction subset. In contrast, the computational expense for exact stochastic simulation scales with the number of reaction events. For an

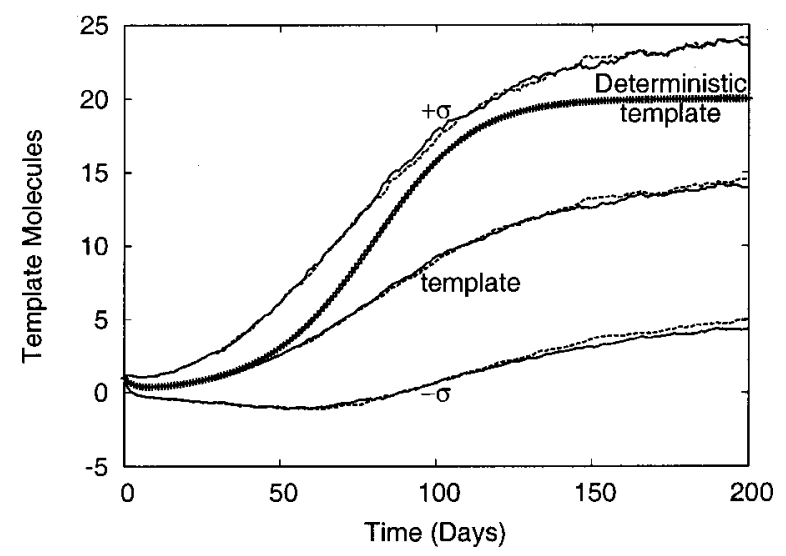

FIG. 8. Comparison of the template mean and standard deviation $( \pm \sigma)$ for exact stochastic (solid lines), approximate stochastic-deterministic (dashed lines), and deterministic (points) simulations. 


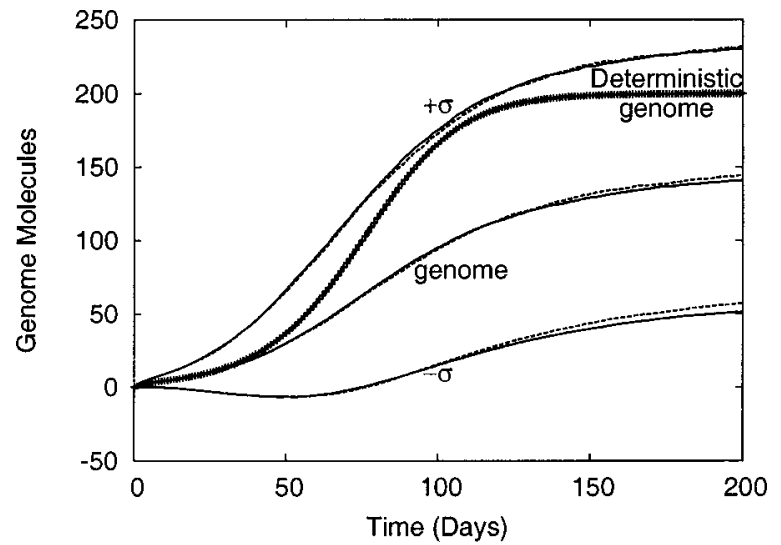

FIG. 9. Comparison of the genome mean and standard deviation $( \pm \sigma)$ for exact stochastic (solid lines), approximate stochastic-deterministic (dashed lines), and deterministic (points) simulations.

example, reconsider simulation of the simple crystallization system presented in Sec. VI A. Doubling the initial amount of A doubles the number of times the fast reaction must occur, and thus significantly increases the computational load of an exact stochastic simulation. On the other hand, if the fast reaction is approximated deterministically, then doubling the initial amount of A does not require stochastic simulation of any additional reaction events, and thus results in no change in the computational load.

The partitioning technique presented in this paper sacrifices some numerical accuracy for a bound on the computational load. Approximating some discrete, molecular reaction events as continuous events via the Langevin approximation loses the discrete nature of the entire system. However, as illustrated by the simple crystallization example, this approximation still accurately reconstructs at least the first two moments of each reacting species. Furthermore, approximating fast reactions deterministically eliminates all fluctuations contributed to the system by these reactions. Depending upon the system and the modeling objective, though, these sacrifices may be acceptable. In the simple crystallization example, the stochastic-deterministic simulations accurately reconstructed the means of all species as well as the standard deviations for the small numbered species. If fluctuations in the larger species are not of interest, then these results are

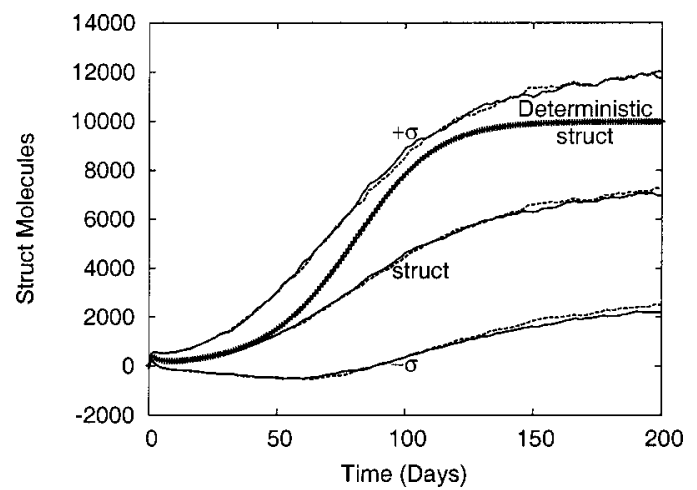

FIG. 10. Comparison of the structural protein (struct) mean and standard deviation $( \pm \sigma)$ for exact stochastic (solid lines), approximate stochasticdeterministic (dashed lines), and deterministic (points) simulations.
TABLE IV. Simulation time comparison for the intracellular viral infection example.

\begin{tabular}{llc}
\hline \hline Solution method & System type & Relative CPU time \\
\hline Exact stochastic & Unpartitioned & 51.5 \\
Stochastic-deterministic & Partitioned & 1 \\
\hline \hline
\end{tabular}

acceptable. In the intracellular viral infection example, the approximate stochastic-deterministic simulation accurately reconstructed the evolution of the probability distribution for the small numbered species, as well as the means and standard deviations for the large numbered species. Here, amplification of fluctuations from the small to large numbered species (template to struct) led to accurate estimates of the statistics of the large numbered species.

A secondary contribution of this paper is an approximate simulation for master equations subject to time-varying constraints. As demonstrated by the simple crystallization example, this approximate simulation approaches the accuracy of the exact simulation as the magnitude of the propensity of no reaction increases. This approximation is most useful for cases in which the total reaction rate, $r_{\text {tot }}$, is not integrable analytically. For this case, we must use an ODE solver with a stopping criterion to determine the next reaction time. Since calling such an ODE solver requires some "overhead" computational expense, performing the approximate simulation may be computationally favorable.

We envision that the primary benefit of the tools presented in this work is bridging the gap from the microscopic to the macroscopic. In particular, researchers are becoming increasingly interested in modeling nanomaterials, phenomena at interfaces, and site interactions on catalysts. In each of these problems, macroscopic interactions in the bulk influence microscopic interactions at interfaces. Although most of the action is at the interface, we cannot neglect the bulk or we lose the ability to model the effect of process design and control strategies. The techniques presented here provide one method of modeling these interactions.

\section{ACKNOWLEDGMENTS}

The authors gratefully acknowledge the financial support of the industrial members of the Texas-Wisconsin Modeling and Control Consortium. This material is based upon work supported under a National Science Foundation Graduate Research Fellowship. All simulations were performed using Octave (http://www.octave.org/). Octave is freely distributed under the terms of the GNU General Public License.

\footnotetext{
${ }^{1}$ A. Arkin, J. Ross, and H. McAdams, Genetics 149, 1633 (1998).

${ }^{2}$ H. McAdams and A. Arkin, Annu. Rev. Biophys. Bioeng. 27, 199 (1998).

${ }^{3}$ I. J. Laurenzi and S. L. Diamond, Biophys. J. 77, 1733 (1999).

${ }^{4}$ D. T. Gillespie, J. Comput. Phys. 22, 403 (1976).

${ }^{5}$ D. T. Gillespie, J. Phys. Chem. 81, 2340 (1977).

${ }^{6}$ J. He, H. Zhang, J. Chen, and Y. Yang, Macromolecules 30, 8010 (1997).

${ }^{7}$ M. A. Gibson and J. Bruck, J. Phys. Chem. A 104, 1876 (2000).

${ }^{8}$ C. Rao and A. Arkin, J. Chem. Phys. (submitted).

${ }^{9}$ J. A. M. Janssen, J. Stat. Phys. 57, 171 (1989).

${ }^{10}$ J. A. M. Janssen, J. Stat. Phys. 57, 187 (1989).
} 
${ }^{11}$ M. O. Vlad and A. Pop, Physica A 155, 276 (1989).

${ }^{12}$ D. T. Gillespie, J. Chem. Phys. 115, 1716 (2001).

${ }^{13}$ H. Resat, H. S. Wiley, and D. A. Dixon, J. Phys. Chem. B 105, 11026 (2001).

${ }^{14}$ Note that reversible reactions can be modeled as two irreversible reactions.

${ }^{15}$ D. T. Gillespie, Physica A 188, 404 (1992).

${ }^{16}$ C. W. Gardiner, Handbook of Stochastic Methods for Physics, Chemistry, and the Natural Sciences, 2nd ed. (Springer-Verlag, Berlin, 1990).

${ }^{17}$ D. T. Gillespie, J. Chem. Phys. 113, 297 (2000).
${ }^{18}$ D. T. Gillespie, Markov Processes: An Introduction for Physical Scientists (Academic, New York, 1992).

${ }^{19}$ Current values of the $r_{k}^{y}$ 's and $r_{\text {tot }}^{y}$ should be available from step (7).

${ }^{20}$ A. P. J. Jansen, Comput. Phys. Commun. 86, 1 (1995).

${ }^{21}$ T. G. Kurtz, J. Chem. Phys. 57, 2976 (1972).

${ }^{22}$ Reactions are partitioned on the basis of the magnitude of their extents, not their rate constants.

${ }^{23}$ T. C. Gard, Introduction to Stochastic Differential Equations (Marcel Dekker, New York, 1988).

${ }^{24}$ R. Srivastava, L. You, J. Summers, and J. Yin, J. Theor. Biol. (to be published). 